\title{
Metodología para diseñar la adaptación de la presentación de contenidos en sistemas hipermedia adaptativos basados en estilos de aprendizaje.
}

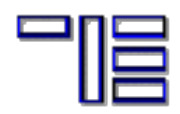

\author{
Marcela Prieto Ferraro \\ mprieto5@usuarios.retecal.es \\ Universidad de Salamanca \\ Helmut Leighton Álvarez \\ hleighton@usuarios.retecal.es \\ Universidad de Salamanca \\ Francisco José García Peñalvo \\ fgarcialusal.es \\ Universidad de Salamanca \\ Begoña Gros Salvat \\ bgrosaub.edu \\ Universidad de Barcelona
}

\section{Introdución}

Los Sistemas Hipermedia (SH) on-line para el aprendizaje presentan, en general, algunas limitaciones importantes. Éstas son básicamente tres, a saber: la desorientación que se produce en algunos estudiantes al recorrer sitios que contienen gran cantidad de información sobre los temas ofrecidos; la fatiga cognitiva que experimentan algunos usuarios al obtener contenidos en diferentes formatos de información y de manera simultánea y, por último, la utilización de una única estrategia instruccional, asumiendo que los estudiantes están dotados de las mismas capacidades, conocimientos, experiencias y estilos para procesar y percibir la información que se les proporciona (Prieto, Gros, García, 2003).

Una alternativa que disminuye el impacto de las limitaciones anteriormente descritas son los Sistemas Hipermedia Adaptativos (SHA), que esencialmente son SH con capacidad de adaptar la presentación de contenidos, los formatos de información y las opciones de navegación a características de sus potenciales usuarios, esto con el fin de proporcionar la información más relevante y en los formatos más adecuados para cada uno de ellos. Particularidades como el nivel de conocimientos, las experiencias previas, el idioma y los intereses fueron inicialmente modeladas para producir la adaptación en los primeros SHA destinados a favorecer el aprendizaje, en un área específica del conocimiento (Prieto, Leighton, García, 2004). Posteriormente, estos tipos de sistemas han incorporado otras variables, los estilos cognitivos o los estilos de aprendizaje de los estudiantes-usuarios. Esta nueva forma de basar la adaptación se fundamenta en que distintos estudiantes no logran un igual grado de desempeño frente a una única forma de enseñanza, que es la que esencialmente proporcionan los $\mathrm{SH}$, sino que requieren de estrategias instruccionales adecuadas a la forma como procesan y perciben la información (Prieto, Gros, García, 2004).

Existe la necesidad de establecer una metodología que permita seleccionar la o las formas de adaptación en función, no sólo de los estilos de aprendizajes o estilos cognitivos, sino también del objetivo de aprendizaje en estos sistemas.

¿Cómo se establecen, a partir de los estilos de aprendizaje las distintas presentaciones de contenidos?, 
¿qué formatos de información es necesario incluir?, ¿qué secuencia establecer entre contenidos, ejemplos y ejercicios?, ¿qué herramientas complementarias se deben incorporar?¿qué técnicas de adaptación de presentación de contenidos permiten generar las presentaciones correspondientes?, son algunas interrogantes que surgen al analizar estos sistemas.

Por este motivo, el objetivo de este trabajo es describir y analizar algunos de los Sistemas Hipermedia Adaptativos Educativos (SHAE) basados en Estilos de Aprendizaje que se han desarrollado y realizar una propuesta metodológica a fin de diseñar cursos on line adaptativos que sean efectivos desde una perspectiva pedagógica. Para ello, se analizan en la sección 2 los SHAE que se han basados en Estilos de Aprendizaje. En la sección 3 se describen las etapas del diseño y de qué manera se relacionan entre sí. En la sección 4 se ejemplifica la propuesta a través de un objetivo de aprendizaje y considerando dos estilos de aprendizaje del modelo de Alonso, Gallego y Honey (1997): Activo y Reflexivo. Finalmente, se establecen las conclusiones en la sección 5.

\section{SHAE basados en Estilos de Aprendizaje}

\subsection{Estilos de Aprendizaje}

Existen en la literatura diversos modelos y teorías de estilos de aprendizaje, donde éstos se definen y clasifican de distintas maneras. Es así como también encontramos diferentes definiciones sobre este concepto, que no son comunes para autores diferentes. Para los efectos de este trabajo consideraremos la dada por Jonassen \& Grabowski (1993) que los definen como las preferencias en la manera de percibir y procesar la información en ambientes de aprendizaje, que son manifestadas por los estudiantes a través de sus respuestas a los inventarios diseñados para ello. El o los estilos preponderantes de los estudiantes, en los distintos modelos, tienen asociados un número de características que los distinguen de otra.

\subsection{Algunos ejemplos de SHAE basados en Estilos de Aprendizaje}

Los SHAE desarrollados y que basan la adaptación en los estilos de aprendizaje de sus potenciales usuarios, se han sustentado en diferentes modelos o teorías de estilos de aprendizaje.

En algunos sistemas la adaptación se materializa exclusivamente en la presentación de los contenidos, en otros, se incorporan además sugerencias para las opciones de navegación. Distintas y variadas estrategias instruccionales, que incluyen distintos formatos de información, organización de contenidos y secuencias de actividades, son utilizadas para ajustar los sistemas a las necesidades de los estudiantes-usuarios en función de sus respectivos estilos de aprendizaje.

Algunos sistemas que adaptan sólo la presentación de los contenidos son: CS383 que es un curso básico de Ciencia de la Computación que introduce a los estudiantes en distintos tópicos y que se basa en los estilos de aprendizaje planteados por Felder-Silverman (Carver, Howard, Lane, 1999). ARTHUR considera los estilos de aprendizaje Kinestésico, Visual o Auditivo (Sarasin, 1998), proporcionando a los estudiantes con distintos estilos información en formatos diferentes (Gilbert \& Han, 1999). El sistema iWeaver (Wolf, 2002) compone distintas páginas para diferentes estudiantes, en función de algunos de los estilos de aprendizaje de Dunn \& Dunn.

Por otra parte hay sistemas en que se adapta además las opciones de navegación. En el caso de INSPIRE los estilos de aprendizaje Activo, Reflexivo, Teórico y Pragmático (Alonso et al., 1997) determinan la adaptación de la presentación de contenidos; la adaptación de las opciones de navegación se determina en relación con el nivel de desempeño (Papanikolaou, Grigoriadou, Magoulas, Kornilakis, 2002). En otros sistemas como MAS PLANG (Peña, Marzo, de la Rosa, Fabregat, 2002), FEIJOO.NET (Paule, Pérez, Pérez, González, 2003) y web-based courseware (Bajraktarevic, May, Fullick, 2003) se adapta la presentación de los contenidos y las opciones de navegación a distintos estilos de aprendizaje del modelo de Felder-Silverman, Activo y Teórico (Alonso et al., 1997) y Global y Secuencial (Felder, 1996) respectivamente. 
En los sistemas que han sido analizados la mayoría de ellos no utilizó, o no hacen explícitos, un conjunto de criterios para la selección de un determinado modelo o teoría de estilos de aprendizaje, excepto los de validez empírica del modelo. Al mismo tiempo es importante destacar que la selección de las estrategias de enseñanza, en los sistemas descritos, está basada exclusivamente en los posibles estilos de aprendizajes de los futuros usuarios, sin considerar otros factores de carácter pedagógico determinantes para un diseño instruccional.

\section{Metodología para diseñar la adaptación de la presentación de contenidos}

\subsection{Criterios para seleccionar la teoría de Estilos de Aprendizaje}

Karagiannidis \& Sampson (2004) plantean un conjunto de criterios útiles para seleccionar el modelo o teoría de estilos de aprendizaje más adecuado para ser incorporado como referencia de la adaptación, estos son: la justificación tanto teórica como empírica del modelo, si posee instrumentos para clasificar a los estudiantes en las categorías que establece, si éstos últimos no son demasiado extensos, si describe además de las categorías de estilos las estrategias instruccionales asociadas a cada una, el costo del instrumento de medición de los estilos y, finalmente, si es apropiado para el contexto de aprendizaje. La aplicación de criterios de orden práctico y, principalmente, pedagógicos es necesaria para escoger el modelo de estilos de aprendizaje más adecuado para el SHAE que será diseñado.

\subsection{Adaptar el proceso instructivo en función del Objetivo y Estilos de Aprendizaje}

Una de las maneras de adaptar el proceso instruccional es ajustándolo a los rasgos individuales o preferencias de cada estudiante, de tal forma de enseñar a los estudiantes utilizando métodos instruccionales que se relacionan con sus fortalezas y preferencias (Jonassen \& Grabowski, 1993).

Si se adopta la modalidad de adaptación a las preferencias individuales, como los estilos de aprendizaje, de acuerdo a Merrill (2000), primero hay que seleccionar las estrategias instruccionales apropiadas y consistentes con el objetivo de aprendizaje y, sobre la base de estas estrategias, optar por las más adecuadas a cada uno de los estilos de aprendizaje Esto permitirá definir las distintas alternativas de presentación de un mismo contenido para cada uno de los estilos de aprendizajes del modelo utilizado para basar la adaptación. Para cada una de estas opciones de presentación de contenidos es necesario establecer los formatos de información que serán utilizados (texto, audio, imágenes, animaciones, vídeo) (Prieto et al., 2004a), las distintas secuencias de los mismos, las herramientas disponibles para las actividades a desarrollar y finalmente, a partir de estos parámetros, las técnicas de adaptación a utilizar para conseguir que un usuario específico, con un estilo de aprendizaje preponderante, acceda a los contenidos adecuados a sus preferencias (Figura $\mathrm{N}^{\circ} 1$ ).

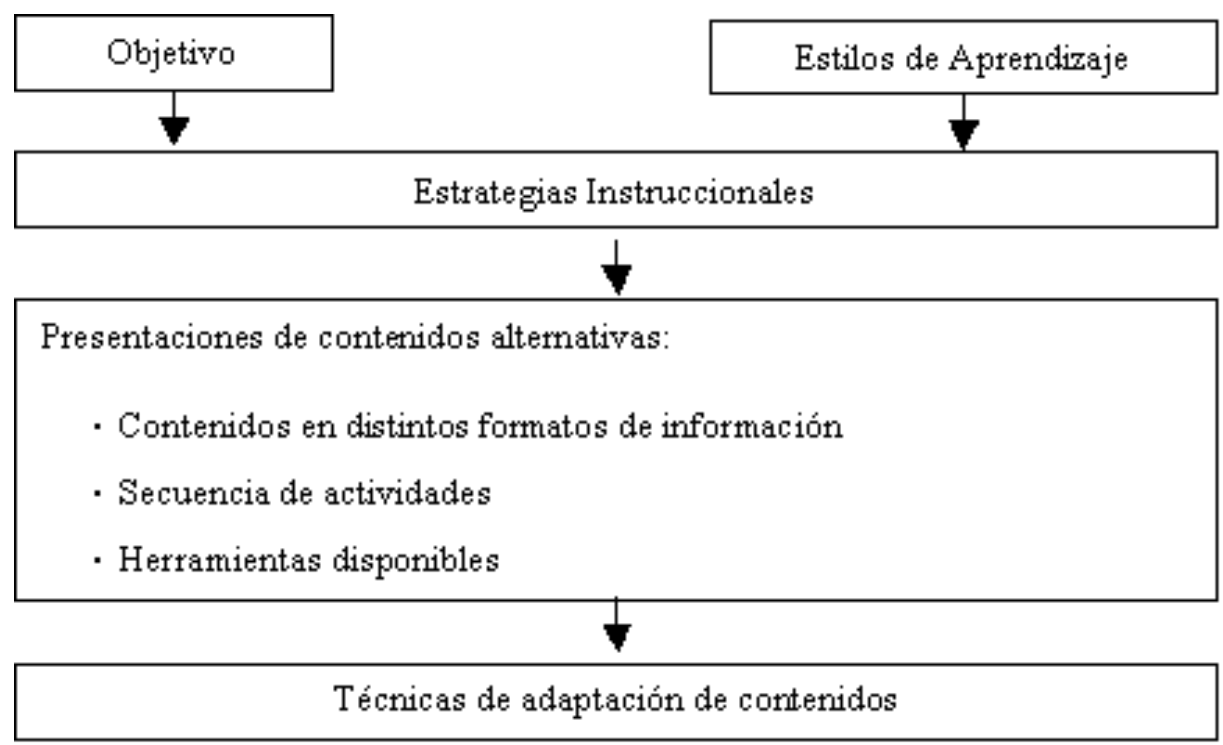


Figura No 1 : Metodología para diseñar la adaptación de la presentación de contenidos.

\subsection{Estrategias instruccionales}

Reigeluth \& Moore (1999) se refieren a distintas estrategias instruccionales utilizadas para facilitar el aprendizaje, clasificándolas de acuerdo a: el tipo de aprendizaje, el control del aprendizaje, la dirección del aprendizaje, el agrupamiento para aprender, las interacciones para aprender y el apoyo para aprender.

- Tipo de aprendizaje se relaciona con el propósito de la actividad y el tipo de aprendizaje afectado (Tabla No 1).

\begin{tabular}{|c|c|}
\hline Memorizar & Aplicación de habilidades \\
\hline Entender relaciones & $\begin{array}{c}\text { Aplicación de destrezas } \\
\text { generales }\end{array}$ \\
\hline
\end{tabular}

Tabla $\mathrm{N}^{\mathrm{o}}$ 1: Tipo de aprendizaje

Aunque estas categorías son distintas entre sí, pueden solaparse y presentar un continuo, por ejemplo que sea necesario que los alumnos requieran memorizar determinada información para aplicar una técnica, o dentro de una perspectiva constructivista actividades del ámbito de las técnicas de orden superior para resolver problemas que incluyen el análisis, la síntesis y la evaluación.

- Control del aprendizaje: en el diseño tradicional es el profesor el que controla los objetivos educativos, selecciona el contenido, determina las estrategias educativas a utilizar y evalúa el aprendizaje. Sin embargo, las nuevas tendencias centradas en el estudiante, dan mayor responsabilidad a este último para definir sus resultados de aprendizaje y escoger el camino para lograr dichos resultados. De esta forma, se puede presentar un continuo entre el control del aprendizaje centrado en el profesor y centrado en el alumno.

- El foco del aprendizaje es un sistema bidimensional compuesto por dos ejes, donde el eje vertical representa la variación desde el dominio específico hasta la interdisciplinariedad. En el eje horizontal los puntos extremos corresponden a un tópico y a la resolución de problemas. El foco puede estar centrado sobre uno de los ejes o en cualquiera de los cuatro cuadrantes permitiendo diferentes combinaciones,

- Agrupamiento para aprender: la agrupación de los estudiantes para el aprendizaje, puede variar desde el trabajo individual hasta el grupal, pasando por el trabajo en pares o equipos.

- Interacciones para aprender: son los posibles tipos de interacción que pueden facilitar el aprendizaje de los alumnos. Existen dos tipos de interacciones, la interacción con otras personas (estudiante-profesor, estudiante-estudiante, otras) y la interacción con recursos no humanos, como herramientas, información y entornos manipulables.

- Apoyo para aprender: los estudiantes requieren de soporte para su mejoramiento y crecimiento. El soporte cognitivo consiste en todos aquellos elementos que sirven al estudiante en la construcción de su entendimiento y mejoramiento de sus competencias en determinada materia, estos recursos pueden ser material impreso, recursos computacionales, interacción con otras personas, secuencias de acceso a información, realimentación y evaluación entre otros. El soporte emocional consiste en aquellos elementos que dan apoyo a las actitudes, motivación, sentimientos y auto confidencia. Estos soportes pueden tomarse como un plano continuo bidimensional, dependiendo de las características propias del estudiante

\subsection{Técnicas de adaptación de contenidos}

Existen diferentes técnicas de adaptación para la presentación de contenidos que facilitan la generación de presentaciones personalizadas en función de las características de los usuarios consideradas para realizar la adaptación. Brusilovsy (2001) describe distintas técnicas utilizadas para adaptar los contenidos: alteración de fragmentos, atenuación de fragmentos, inserción/eliminación de fragmentos, ordenación de 
fragmentos, técnica basada en marcos, texto expansible, variante de fragmentos y variante de páginas, que son descritas en la Tabla $\mathrm{N}^{\circ} 2$.

\begin{tabular}{|l|l|}
\hline \multicolumn{1}{|c|}{ Técnica } & Características \\
\hline Alteración de fragmentos & $\begin{array}{l}\text { Un mismo fragmento es alterado, agregando o suprimiendo } \\
\text { información }\end{array}$ \\
\hline Atenuación de fragmentos & Ensombrecer trozos de textos que son irrelevantes para un usuario \\
\hline $\begin{array}{l}\text { Inserción/Eliminación de } \\
\text { fragmentos }\end{array}$ & Agregar o remover fragmentos \\
\hline Ordenación de fragmentos & Proporciona los mismos fragmentos ordenados de diferentes maneras \\
\hline Técnica basada en marcos & $\begin{array}{l}\text { Un marco dividido en campos que contienen distintas explicaciones } \\
\text { para un mismo concepto, enlaces o ejemplos }\end{array}$ \\
\hline Texto expansible & Al seleccionar un texto resaltado es reemplazado por otro texto \\
\hline Variantes de fragmentos & Reemplazar el/los fragmentos por otro(s) \\
\hline Variantes de páginas & Reemplazar una página completa por otras \\
\hline
\end{tabular}

Tabla $N^{\mathrm{o}}$ 2: Técnicas de adaptación de presentación de contenidos.

\section{Ejemplo}

Consideremos sólo los estilos de aprendizaje Activo y Reflexivo del modelo de Alonso et al. (1997), para ejemplificar la metodología propuesta anteriormente. Podemos relacionar las características principales de cada uno de estos estilos con las estrategias instruccionales más adecuadas, descritas en la sección 3.3, en función de las preferencias para aprender de los estudiantes con estos estilos predominantes (Tabla $\mathrm{N}^{\mathrm{o}}$ $3)$.

\begin{tabular}{|c|c|c|}
\hline Estilo & Características & Estı \\
\hline Activo & $\begin{array}{l}\text { Intentar cosas nuevas, nuevas experiencias, } \\
\text { nuevas oportunidades } \\
\text { - Competir en equipo, resolver problemas en } \\
\text { equipo, encontrar personas de mentalidad } \\
\text { semejante para dialogar, dirigir debates y } \\
\text { reuniones } \\
\text { Generar ideas sin formalismos ni estructura, } \\
\text { cambiar y variar las cosas, arriesgarse, sentirse } \\
\text { ante un reto con recursos inadecuados, intentar } \\
\text { algo diferente }\end{array}$ & $\begin{array}{l}\text { Control centrado en el } \\
\text { estudiante } \\
\text { - Trabajo interdisciplinario } \\
\text { Trabajo con pares } \\
\text { Interacción con pares }\end{array}$ \\
\hline Reflexivo & $\begin{array}{l}\text { Considerar las experiencias desde distintas } \\
\text { perspectivas } \\
\text { - Recoger datos y analizarlos antes de establecer } \\
\text { conclusiones } \\
\text { - Son pacientes, detallistas, previsores y } \\
\text { estudiosos de comportamientos }\end{array}$ & $\begin{array}{l}\text { Control más centrado en el } \\
\text { profesor. } \\
\text { Trabajo orientado al } \\
\text { dominio y tópico. } \\
\text { - Trabajo individual más que } \\
\text { grupal. } \\
\text { Interacción con material } \\
\text { impreso, audiovisual. }\end{array}$ \\
\hline
\end{tabular}

Tabla $N^{o}$ 3: Características de los estilos de aprendizaje Activo y Reflexivo y estrategias instruccionales asociadas. 
Consideremos un objetivo de aprendizaje.

- Objetivo: los estudiantes serán capaces de identificar el nombre y la ubicación de partes de un dispositivo o sistema.

De acuerdo al objetivo señalado y a las características de los estilos Activo y Reflexivo, podemos establecer las estrategias instruccionales necesarias para facilitar el logro del objetivo.

\section{- Estrategias instruccionales:}

i. De acuerdo al objetivo: para este tipo de aprendizaje Merrill (2000) sugiere estructurar el conocimiento de manera que se ilustre el dispositivo destacando la ubicación, identificación y la función o propósito de cada componente; presentar los contenidos al estudiante mostrando las distintas partes del sistema, permitiendo realizar exploraciones de las mismas, esto puede lograrse mediante el uso de pop-up menus cuando el estudiante señale cada componente o mediante una presentación que destaque cada parte indicando el nombre y una descripción.

\section{ii. Para el estilo Activo:}

- Permitir al estudiante establecer la secuencia al revisar los contenidos.

- Entregarle una visión de los contenidos desde distintas perspectivas disciplinarias.

- Proporcionar herramientas que permitan el trabajo con un par, en grupos o equipos.

\section{iii. Para el estilo Reflexivo:}

- Incluir información tanto en formato textual como de imágenes y animaciones.

- Establecer las actividades en una secuencia que incluya en primer lugar la revisión de los contenidos y luego el desarrollo de ejercicios.

- Proporcionar documentos anexos en distintos formatos de información..

- Presentación de contenidos: de acuerdo a las estrategias descritas, para cada estilo debería incluir:

i. Para el estilo Activo:

- Incluir información tanto en formato textual como de imágenes incluyendo menús de ayuda para la descripción de cada componente del sistema.

- Establecer las distintas actividades (revisión de contenidos, ejercicios y proyectos colaborativos), de manera que el estudiante escoja libremente la secuencia.

- Proporcionarle herramientas que inviten a trabajar de manera conjunta con sus pares, por ejemplo, pizarras compartidas, chat o foros.

\section{ii. Para el estilo Reflexivo:}

- Incluir información tanto en formato textual como de imágenes y animaciones, menús de ayuda para la descripción de cada componente del sistema.

- Las actividades tendrán una secuencia que incluya la revisión de los contenidos y luego el desarrollo de ejercicios.

- Proporcionar documentos anexos complementarios que contengan distintos formatos de información. 
Técnicas de adaptación que permiten concretar estas distintas presentaciones de contenidos son:

i. $\quad$ Texto expansible: al indicar una parte del dispositivo se despliega información de dicha componente relativa a las características y funcionalidad. Esta técnica permite aplicar la estrategia instruccional sugerida, en función del tipo de objetivo de aprendizaje (Merrill, 2000). Sin embargo, aunque esta técnica es aplicable en ambas presentaciones de contenidos, la cantidad y el tipo de información a desplegar es diferente para cada estilo.

ii. Variantes de páginas: reemplazar una página completa por otra, de manera que para el caso del estudiante con estilo Activo estén disponibles los enlaces que le permitan recorrer la información libremente y acceder a herramientas para trabajar con otros estudiantes; por el contrario, en el caso del estudiante con un estilo reflexivo los enlaces sólo deben permitir la secuencia establecida anteriormente y el acceso a material complementario. Esta técnica permite proporcionar una presentación de contenidos adecuada a las necesidades y preferencias de estudiantes con estilos de aprendizaje distintos.

El creciente desarrollo de $\mathrm{SH}$ destinados a favorecer distintos tipos de aprendizaje, con gran cantidad de información y utilizando los más diversos medios, tanto de manera formal como informal, a través de la red Internet y el cada vez mayor acceso masivo a estos recursos, con usuarios de distinta procedencia cultural, lenguaje, edades, conocimientos, experiencias, intereses y preferencias hace necesario diseñar sistemas con capacidad de adaptación. Esto con la finalidad de que sea el sistema el que se adapte a los potenciales estudiantes y no a la inversa.

El diseño de SHAE involucra diversos y complejos aspectos, tanto de tipo tecnológico, informáticos como pedagógicos. Para generar la adaptación en un SH, basada en una o más características de los potenciales usuarios, requiere de la aplicación de técnicas tanto para la presentación de contenidos como para las opciones de navegación.

En esta propuesta se sugieren diferentes etapas para seleccionar solamente las técnicas de adaptación de la presentación de contenidos.

Propusimos una metodología para diseñar SHAE basados en estilos de aprendizaje enfatizando los aspectos relativos al diseño instructivo, de manera de desarrollar sistemas que efectivamente faciliten el logro de los objetivos de aprendizaje, tomando en cuenta el objetivo de aprendizaje, los estilos de aprendizaje descritos en un modelo concreto y las estrategias instruccionales asociadas.

Los aspectos aquí considerados están basados en los estilos Activo y Reflexivo planteados por (Alonso et al., 1997). Sin embargo, esta misma metodología es válida para diseñar las presentaciones de contenidos de los demás estilos de la misma teoría o de otra en que el SHAE se base.

\section{Agradecimientos}

Deseamos manifestar nuestro agradecimiento al grupo AWEG -Adaptive Web Engineering Group - de la Universidad de Salamanca por sus contribuciones e ideas en el desarrollo de este trabajo. Este trabajo ha sido parcialmente financiado por el proyecto ODISEAME (Open distance Interuniversity Synergies between Europe, Africa and Middle East), ref. EUMEDIS B7-41100/2000/2165-79 P546.

\section{Bibliografía}

AlONSO, C., GAlleGO, D., HONEY, P. (1997). Los Estilos de Aprendizaje. Procedimientos de diagnóstico y mejora. Bilbao: Ediciones Mensajero.

BAJRAKTAREVIC, N., HALL, W., FULLICK, P. (2003). Incorporating learning styles in hypermedia environment: Empirical evaluation. En: Proc. of AH2003 workshop at 12th World Wide Web Conference, Budapest, Hungary, 41-52. 
Brusilovsky, P. (2001). Adaptive Hypermedia. User Modeling and User Adapted Interaction, Vol. 11 (1/2), 87-110.

CARVER,C. A., HOWARD, R. A., LANE, W.D. (1999). Addressing Different Learning Styles Through Course Hypermedia. IEEE Transactions on Education 42(1), 33-38.

FELDER, R.M. (1996). Matters of Style. ASEE Prism, 6(4), 18-23.

GILBERT, J. E. \& HAN, C. Y. (1999). Adapting Instruction in Search of "A Significant Difference". Network and Computer Applications, 22, 149-160.

JONASSEN, D. \& GRABOWSKI, B. (1993). Handbook of individual differences. Hillsdale, NJ: Lawrence Erlbaum Associates

KARAGIANNIDIS, CH. \& SAMPSON, D. (2004). Adaptation rules relating learning styles research and learning objects meta-data. En L. Arroyo \& C. Tasso (Eds.): AH2004 Workshop Proceedings. Part I. Eindhoven: TU/e.

MERRILL, M.D. (2000). Instructional Strategies and Learning Styles: Which takes Precedence?. En Robert Reiser and Jack Dempsey (Eds.) Trends and Issues in Instructional Technology. Prentice Hall.

PAPANIKOLAOU, K., GRIGORIADOU, M., MAGOULAS, G., KORNILAKIS, H. (2002). Towards new forms of knowledge communication: the adaptive dimension of a web-based learning environment. Computers and Education, 39, 333-360.

PAUlE, M., PÉREZ, P., PÉREZ, J., GONZÁLEZ, M. (2003). Feijoo.net: An Approach to Personalized E-learning Using Learning Styles. ICWE 2003, 112-115

PEÑA, C., MARZO, J., DE LA ROSA, J., FABREGAT, R. (2002). Un sistema de tutoría inteligente adaptativo considerando estilos de aprendizaje. IE2002, Vigo, España, 20-22.

PRIETO, M., GROS, B., GARCÍA, F.J. (2003). Modelos para la Elaboración de Materiales Hipermedia Adaptativos para el Aprendizaje. Informe Técnico (DPTOIA-IT-2003-003), Departamento de Informática y Automática, Universidad de Salamanca (España). http://tejo.usal.es/inftec/2003/DPTOIAIT-2003-003.pdf (2003)

PRIETO, M., GROS, B., GARCÍA, F.J. (2004a). Técnicas de adaptación de presentación de contenidos en sistemas hipermedia adaptativos basados en estilos de aprendizaje. En: J.M Sánchez, J.A. Gómez, M. A. Vega, B. Fernández, J. Bravo (Eds.): VI Simposio Internacional de Informática Educativa (SIIE 2004) Avances en Informática Educativa. Cáceres: Universidad de Extremadura.

PRIETO, M., LEIGHTON, H., GARCÍA, F.J. (2004b). Adaptive Educational Hypermedia Proposal based on Learning Styles and Quality Evaluation. En: W. Nejdl \& P. De Bra (Eds.): AH2004, Adaptive Hypermedia and Adaptive Web-Based Systems, LNCS 3137, pp. 316-319, 2004. Berlin: Springer-Verlag.

REIGELUTH, C. M. Y MOORE, J. (1999). Cognitive Education and the Cognitive Domain. In Reigeluth, C. M. (Eds) A New Paradigm of Instructional Theory - Vol. II,.Lawrence Erlbaum Associates, Inc, Publishers (LEA) USA 51-68

SARASIN, L. C. (1998). Learning style perspectives: Impact in the classroom. Madison, WI: Atwood.

WOLF, C. (2002). iWeaver: Towards an Interactive Web-Based Adaptive Learning Environment to Address Individual Learning Styles. EURODL 2002. Disponible en: http://www.adaptivelearning.net/media/html/iWeaver.htm 
(C) Ediciones Universidad de Salamanca 